\title{
Mechanisms and outcomes of the supercharged end-to-side nerve transfer: a review of preclinical and clinical studies
}

\author{
Nicholas von Guionneau, MBBS, ${ }^{1,3}$ Karim A. Sarhane, MD, MSc, ${ }^{1}$ Gerald Brandacher, MD, ${ }^{1}$ \\ Shehan Hettiaratchy, MA, DM, ${ }^{4}$ Allan J. Belzberg, MD, ${ }^{2}$ and Sami Tuffaha, MD ${ }^{1}$ \\ Departments of ${ }^{1}$ Plastic and Reconstructive Surgery and ${ }^{2}$ Neurosurgery, Johns Hopkins University School of Medicine, \\ Baltimore, Maryland; ${ }^{3}$ Department of Brain Sciences, Imperial College London; and ${ }^{4}$ Department of Plastic and Reconstructive \\ Surgery, Imperial College Healthcare NHS Trust, St Mary's Hospital, London, United Kingdom
}

\begin{abstract}
Proximal peripheral nerve injuries often result in poor functional outcomes, chiefly because of the long time period between injury and the reinnervation of distal targets, which leads to muscle and Schwann cell atrophy. The supercharged end-to-side (SETS) nerve transfer is a recent technical innovation that introduces donor axons distally into the side of an injured nerve to rapidly innervate and support end organs while allowing for additional reinnervation after a proximal repair at the injury site. However, the mechanisms by which donor axons grow within the recipient nerve, contribute to muscle function, and impact the regeneration of native recipient axons are poorly understood. This uncertainty has slowed the transfer's clinical adoption. The primary objective of this article is to comprehensively review the mechanisms underpinning axonal regeneration and functional recovery after a SETS nerve transfer. A secondary objective is to report current clinical applications in the upper limb and their functional outcomes. The authors also propose directions for future research with the aim of maximizing the clinical utility of the SETS transfer for peripheral nerve surgeons and their patients.
\end{abstract}

https://thejns.org/doi/abs/10.3171/2020.3.JNS191429

KEYWORDS peripheral nerve; nerve injury; nerve transfers; supercharged end-to-side; regeneration; mechanisms

$\mathrm{P}$ ERIPHERAL nerve injuries (PNIs) often result in poor functional outcomes despite our best efforts at surgical repair and rehabilitation. Among the many factors that influence PNI prognosis, including age and comorbidities, the amount of time that elapses prior to end-organ reinnervation is the most consequential. ${ }^{1}$ This is evidenced by the poor outcomes that can be expected following delayed PNI repairs and proximal repairs in which axons must travel long distances relative to their slow rate of regeneration. Beginning at the time of injury, denervated muscle undergoes progressive, irreversible atrophy that limits the extent to which motor function can be restored when reinnervation occurs. ${ }^{2,3}$ Additionally, proliferating Schwann cells within the distal nerve that lack axonal contact progressively lose the capacity to support regenerating axons, which in turn diminishes and further delays reinnervation and functional recovery. ${ }^{4}$

Nerve transfers have emerged as an approach to achieve faster reinnervation of distal targets and thereby limit the deleterious effects of prolonged denervation. They are performed distal to the injury site in the setting of proximal PNI and shorten the regenerative distance and time needed for reinnervation. The clinical application of end-to-end (ETE) nerve transfers for this purpose has rapidly expanded to a multitude of clinical scenarios with comparable or superior results relative to those following primary repair of proximal nerve injuries. ${ }^{5}$ End-to-side (ETS) transfers, in which the transected end of a distal nerve stump is coapted to the side of an intact donor nerve, have been successfully employed as an alternative to ETE transfers that avoids the morbidity associated with donor nerve transection, although fewer regenerating axons are introduced into the recipient nerve (Fig. 1 left). The supercharged end-to-side (SETS) transfer is a more recent technical innovation in which the proximal end of a donor nerve is sutured to a connective tissue window in the side of an injured recipi-

ABBREVIATIONS AIN = anterior interosseus nerve; ETE = end-to-end; ETS = end-to-side; PNI = peripheral nerve injury; SETS = supercharged end-to-side. SUBMITTED May 22, 2019. ACCEPTED March 30, 2020. 


\section{Traditional ETS}

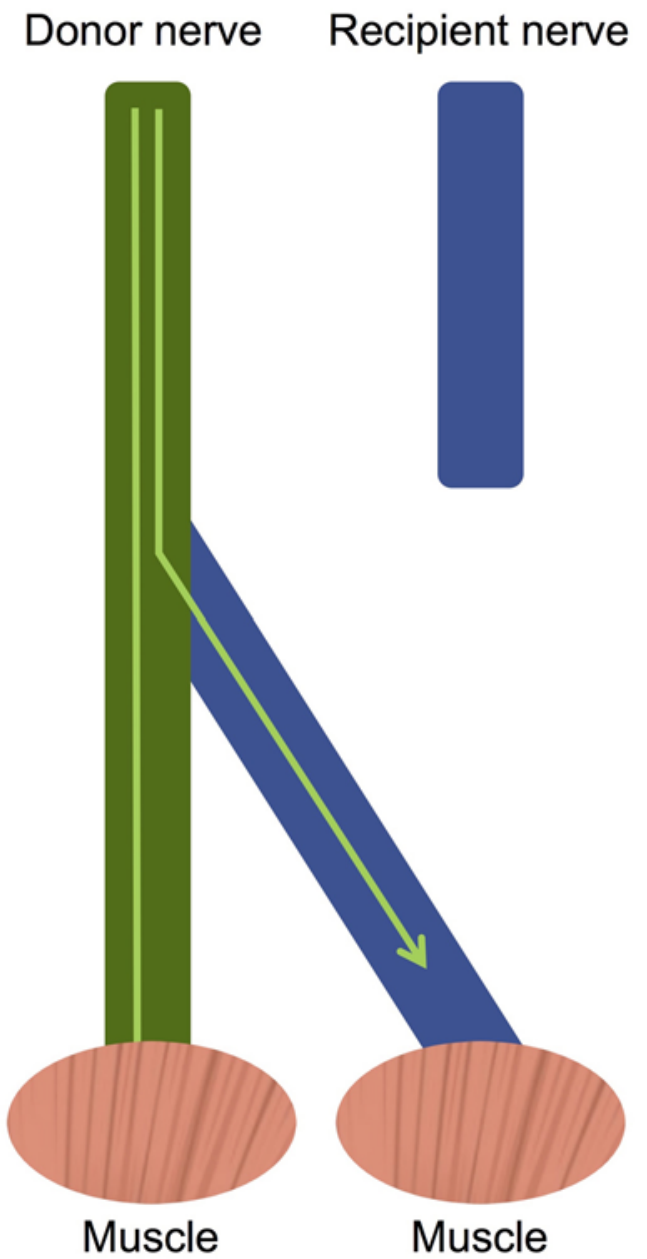

\section{Supercharged ETS}

Donor nerve Recipient nerve

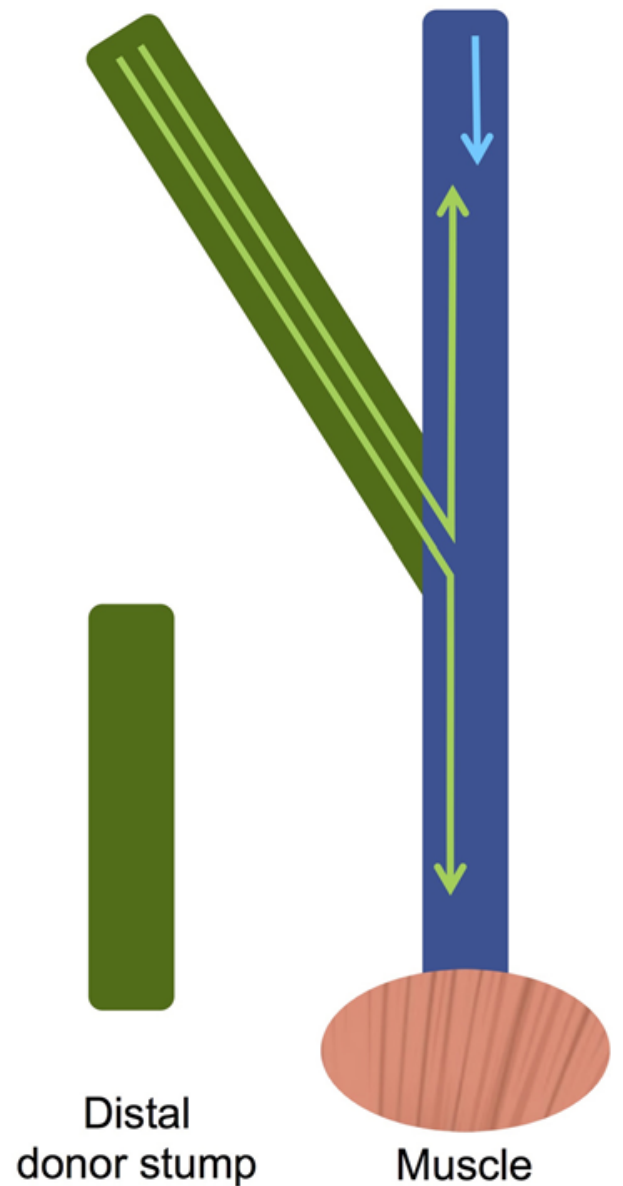

FIG. 1. Anatomy of traditional ETS compared to SETS nerve transfer. Arrows indicate regenerating axons. The SETS transfer reverses donor and recipient roles of the traditional ETS transfer. Left: Traditional ETS coaptation requires transection of the recipient nerve and coaptation of the distal denervated stump into the side of an intact donor nerve; any axons present in the distal recipient nerve stump originate exclusively from the donor nerve. Right: SETS coaptation involves transection of the donor nerve and coaptation of the proximal stump into an injured recipient nerve; axons present in the distal recipient nerve may originate from either donor or recipient nerves. Figure is available in color online only.

ent nerve (Fig. 1 right). ${ }^{6}$ As with ETE and ETS transfers, the SETS transfer is indicated in proximal nerve injuries as a way of introducing axons distally into the injured nerve to rapidly innervate and support end organs. By avoiding recipient nerve transection, the SETS transfer offers the advantage of allowing additional reinnervation from a proximal repair at the injury site and can therefore also be thought of as a "babysitting" procedure (Table 1).

The principle of SETS transfers has great potential to improve functional recovery after proximal nerve injuries. However, a poor understanding of the transfer's mechanisms has slowed its clinical adoption. ${ }^{6-9}$ This article aims to categorize and evaluate those mechanisms in order to provide a framework to improve its clinical translation. We review the underlying mechanisms, clinical indications, and functional outcomes of the SETS transfer.

\section{Methods}

A search was performed using the PubMed database and the terms "(Nerve) AND ((reverse end*) OR (supercharg*))" for all years up to and including April 2019. Animal studies focusing on mechanisms underlying nerve regeneration and functional outcomes after SETS transfer were reviewed. We also used clinical reports of SETS transfers after proximal ulnar nerve injury. Additional records were identified by examining references of relevant studies and reviews.

Of note, there is a lack of consensus on the nomenclature for the SETS transfer. ${ }^{7}$ In this review, we use the term "supercharged end-to-side transfer" (rather than "reverse end-to-side," "supercharge," or "supercharging"), and we recommend this term to consolidate the field going forward. 
TABLE 1. Pros and cons of different nerve transfers

\begin{tabular}{lccc}
\hline \multirow{2}{*}{ Factor } & \multicolumn{3}{c}{ Transfer Type } \\
\cline { 2 - 5 } & ETE & ETS & SETS \\
\hline Requires donor nerve sacrifice & $\checkmark$ & x & $\checkmark$ \\
\hline $\begin{array}{l}\text { Allows for regeneration from proximal recipient } \\
\text { nerve repair site }\end{array}$ & x & x & $\checkmark$ \\
\hline Augments existing recipient nerve function & x & x & $\checkmark$ \\
\hline $\begin{array}{l}\text { Can "babysit" end organ w/o needing a 2nd } \\
\text { procedure }\end{array}$ & x & x & $\checkmark$ \\
\hline
\end{tabular}

\section{Mechanisms Underlying Nerve Regeneration in the SETS Transfer \\ Overview of the SETS Growth Environment}

The growth stimuli associated with axonal regeneration across a SETS transfer differ from those present in a traditional ETS transfer (Fig. 2). In a SETS coaptation, the donor nerve is transected and all donor axons will attempt to regenerate into the recipient nerve. By contrast, in ETS transfers, the majority of donor axons remain undamaged while roughly a third of donor axons regenerate across the coaptation..$^{10,11}$ After both ETS and SETS coaptations, once axons enter the recipient nerve, they are subject to a similar regenerative environment. However, in SETS transfers, native recipient axons may also regenerate into the distal recipient nerve. The impact of donor axons on native axonal growth is unclear.

\section{Penetration of Donor Axons Into Recipient Nerve: Epineurial Versus Perineurial Window}

The degree of connective tissue disruption in the recipient nerve at the site of a SETS coaptation that facilitates maximal regeneration across the coaptation is controversial. ${ }^{7,12}$ Preclinical models have demonstrated robust axonal regeneration across the SETS coaptation with an epineurial window ${ }^{6,13-16}$ or a perineurial window. ${ }^{9,17}$ The presence of donor axons in a recipient nerve distal to the SETS coaptation has been confirmed histologically using light microscopy, ${ }^{6}$ immunofluorescence, ${ }^{9,14}$ and retrograde labeling. ${ }^{13,14,16}$

In rodent models, the difficulty distinguishing epineurial from perineurial windows adds to the challenge of assessing the relative benefit of either window in the SETS transfer. The two tissue layers are often confluent, and performing a perineurial coaptation is technically challenging. Only one of five research groups has reported using a perineurial window in preclinical studies., ${ }^{9,17}$ That group found a much higher myelinated axon count with a perineurial window (3127 axons, "Group 3 RETS") $)^{9}$ than Nadi et al. did after SETS transfer with an epineurial window (745 axons, "Group 4 SETS control")..$^{16}$ Animals in both of these experimental subgroups were subject to the same experimental conditions. No comparable functional data were available. This limited comparison offers weak evidence that a perineurial window may allow greater donor axon regeneration into the recipient nerve.

Perineurial trauma is known to negatively impact axon regeneration. Gordon et al. demonstrated this in a crossbridge nerve graft model in which longitudinal perineurial stripping of a recipient nerve was associated with less donor motor neuron regeneration as well as less native recipient axon regeneration after delayed repair than the use of a perineurial window. ${ }^{10}$ Minimizing perineurial trauma at the site of a SETS coaptation may improve donor axon penetration into the recipient nerve.

Clinical applications of the SETS coaptation involving anterior interosseous nerve (AIN) to ulnar nerve transfer used perineurial windows, $, 8,9,18$ although this approach is based on findings of enhanced regenerative outcomes after more extensive donor nerve injury in traditional ETS models. ${ }^{12}$ This rationale may not apply to a SETS coaptation in which the connective tissue window is meant to facilitate axon penetration into the recipient nerve rather than out of the donor nerve. Whether the depth of the connective tissue window improves the penetration of donor axons into the recipient nerve in a SETS coaptation has yet to be examined. A possible drawback of opening the recipient perineurium in a SETS transfer performed immediately after proximal injury is that it may recreate an axotomy injury and inadvertently allow for ETE repair between donor and recipient nerves. This could negatively impact the potential for native recipient axon regeneration. Ultimately, the optimal window depth should balance the hypothesized benefits of improved donor axon penetration into the recipient nerve with the cost of damaging the recipient nerve structure and the potential for native regeneration.

\section{Growth Within the Denervated Nerve: Direction of Donor Axon Growth}

The use of Thyl-GFP rats demonstrated that donor axons regenerate in both proximal and distal directions within the recipient nerve after crossing a SETS coaptation. ${ }^{9,19}$ These experiments also revealed the speed of axonal growth across a SETS coaptation: penetration into a recipient rat tibial nerve by day 7 and diffuse spread across the cross-sectional area of the nerve by day $10 .{ }^{9}$ Evidence from an elegant cross-bridging nerve graft study (in which the distal coaptation is equivalent to a SETS coaptation) by Gordon et al. quantified this bidirectional axonal growth, with the authors finding no difference in axon count or fiber type, proximally versus distally in the recipient nerve. ${ }^{10}$ The results echo the findings of Balik and Menderes who showed an equal axon count and ratio of myelinated to unmyelinated fibers on either side of a modified SETS transfer in which each direction in the recipient nerve ended in a separate muscle. ${ }^{20}$

Isaacs et al. attempted to determine whether donor axons regenerating in a proximal direction after a SETS coaptation become functional motor units. ${ }^{13}$ They measured evoked muscle contraction force after transecting the recipient nerve just proximal to the SETS coaptation in an attempt to eliminate donor axons growing in a proximal direction. There was no significant change in muscle force compared to stimulation after a much more proximal transection of the recipient nerve, suggesting that proximally growing donor axons do not contribute materially to functional motor units. Notably, the standard deviations 
Traditional ETS

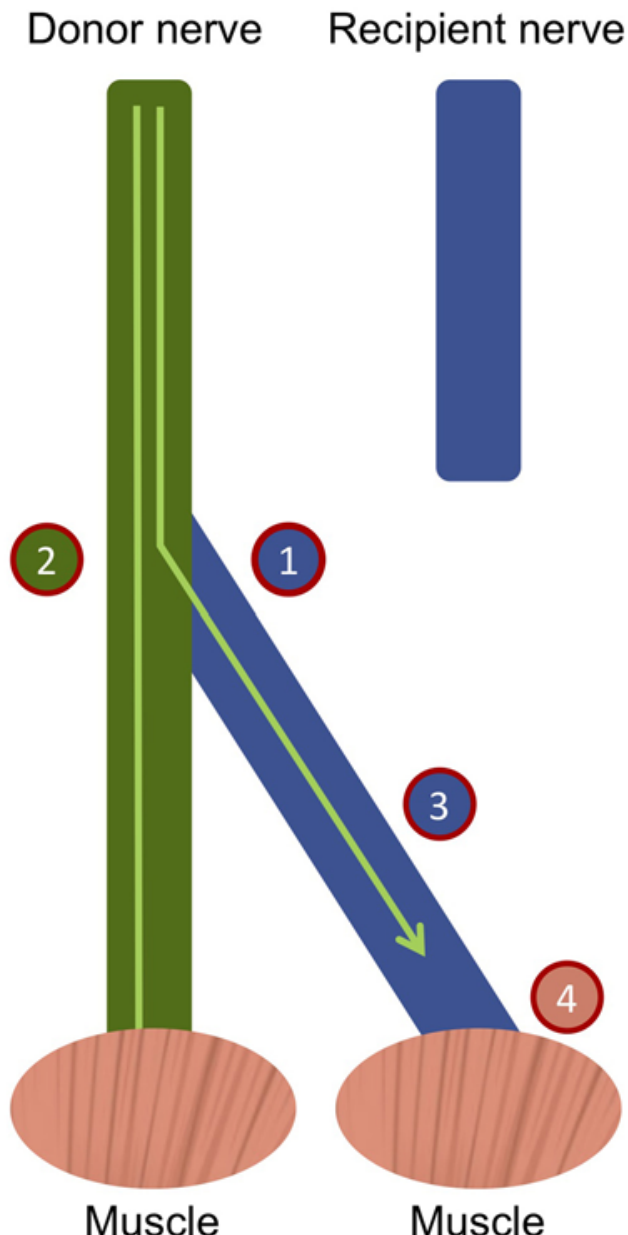

\section{Supercharged ETS}

\section{Donor nerve Recipient nerve}

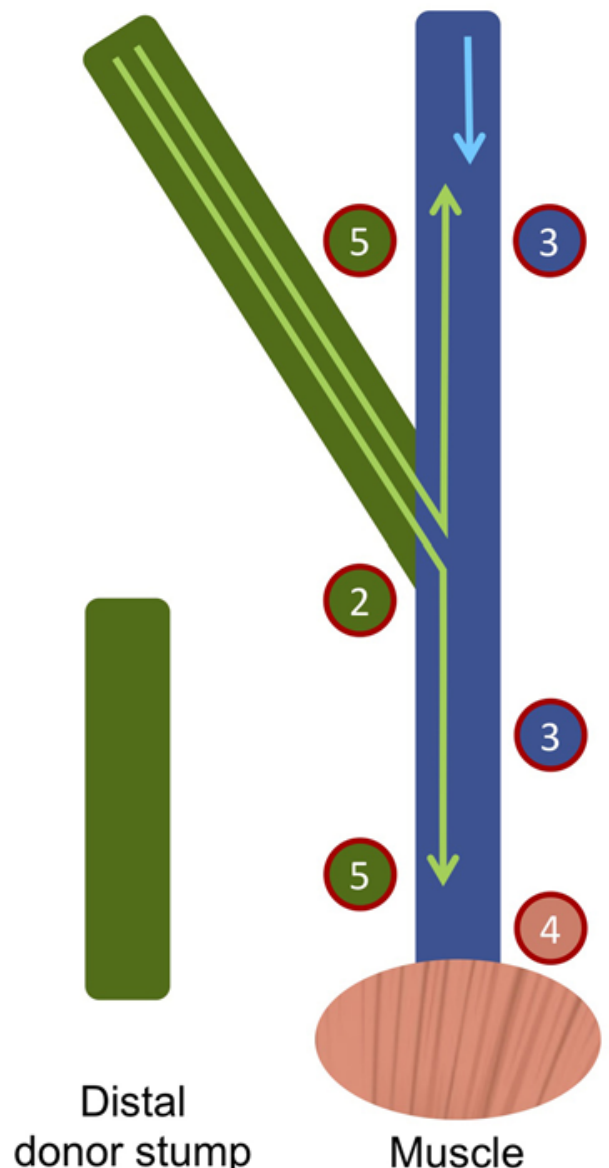

FIG. 2. Factors affecting the regenerative environment after ETS and SETS transfers. The color of each number indicates the source of the regenerative stimulus: green, donor nerve; blue, recipient nerve; pink, muscle. Left: In a traditional ETS transfer, an exposed distal stump is a potent regenerative stimulus attracting donor axons (1); injury to some donor nerve axons caused by opening a connective tissue window or by epineurial sutures promotes regeneration of donor axons (2); denervated Schwann cells establish a proregenerative environment in the recipient nerve (3); muscle-derived trophic support (4). Right: In a SETS transfer, transection of the donor nerve results in growth cone formation in all donor axons (2); denervated Schwann cells establish a proregenerative environment in the recipient nerve proximal and distal to the SETS coaptation site (3); muscle-derived trophic support (4); regenerated donor axons influence regenerating native axons within the recipient nerve (5). Figure is available in color online only.

of their force testing data were large, implying their experimental model may have been unreliable. In summary, donor axons do not appear to grow preferentially in either direction after crossing a SETS coaptation, and there is insufficient evidence to determine the fate and impact of proximally growing donor axons.

\section{Growth Within the Denervated Nerve: Donor Axon Access to Endoneurial Structures}

Essential to functional recovery after PNI is the regeneration of motor neurons within endoneurial tubes to motor end plates. ${ }^{21-23}$ Using light microscopy, Isaacs et al. observed donor axons in the recipient nerve traveling in fascicular groups as well as in the epineurial space. ${ }^{6} \mathrm{Fu}-$ jiwara et al. used immunofluorescent staining of connective tissues to confirm that donor axons penetrate recipient perineurium. ${ }^{14}$

Donor axon access to recipient nerve endoneurial structures may be affected by their direction of growth within the recipient nerve. After perineurial stripping at the coaptation site, Gordon et al. found that donor axons growing proximally traveled in equal numbers inside and outside the perineurium, whereas donor axons growing distally regenerated almost exclusively inside perineurium. ${ }^{10}$ When only a perineurial window was used at the site of the SETS coaptation, the vast majority of donor axons traveling proximally regenerated within perineurial structures. These findings support a hypothesis that limiting damage 
to recipient nerve connective tissues improves donor axon access to subperineurial structures. Perhaps extensive connective tissue disruption in the recipient nerve alters local expression of factors involved in directing axon growth into subperineurial structures, such as tenascin-C. ${ }^{24}$ The findings also raise the possibility that axonal growth toward a denervated end organ may be preferentially directed into subperineurial structures.

The fate of donor axons regenerating outside of endoneurial tubes after crossing a SETS coaptation is unknown. Axonal regeneration outside of native pathways is thought to result in poor muscle function. ${ }^{2}$ However, previous studies have shown that axons that have regenerated through epineurial sheath conduits are capable of reinnervating muscles. ${ }^{25-27}$ Neuroanatomical protocols capable of anterogradely tracing axons could be useful in addressing this question. ${ }^{28}$

\section{Growth Within the Denervated Recipient Nerve: Importance of Schwann Cells}

Chronic Schwann cell denervation, misdirected axonal growth, and muscle atrophy are well known to contribute to poor functional outcomes after nerve injury. ${ }^{29}$ After an acute injury, Schwann cells lose their myelinating phenotype and dedifferentiate to a proliferating repair phenotype capable of phagocytosing degenerated myelin and axonal debris, supporting axonal regrowth and guiding regenerating axons to end-organ targets. ${ }^{30,31}$ Repair-type Schwann cells extend long processes along the inside of denervated endoneurial tubes to form "bands of Büngner" (longitudinal structures that support and direct regenerating axons). However, after prolonged periods without axonal contact, Schwann cells atrophy, losing the ability to maintain bands of Büngner ${ }^{32-34}$ and to upregulate nerve growth factors. ${ }^{35-37}$

Beyond babysitting muscle, the SETS transfer can be used to more quickly reintroduce axons to denervated Schwann cells in the distal recipient stump after injury and thereby mitigate the negative effects of chronic denervation on these critical support cells. Hendry et al.'s sideto-side nerve bridge model (in which distal coaptation is equivalent to a SETS transfer) successfully demonstrated that the prompt introduction of donor axons into a denervated recipient nerve via a SETS coaptation can prevent chronic denervation of Schwann cells. ${ }^{38}$ Contact with regenerating axons induces a second round of Schwann cell proliferation with upregulation of growth-promoting transcription factors, neurotrophins, and growth factor receptors $^{38-41}$ that may benefit both donor and native recipient axon regeneration.

\section{Native Axon Regeneration After the SETS Transfer}

The addition of a SETS transfer has been shown to increase the total number of axons in the distal recipient nerve compared to the number of axons present after ETE repair and crush injury without SETS protection. ${ }^{16,42,43}$ However, the impact of donor axons on native axon regeneration is poorly understood.

Two studies have used double retrograde labeling to determine the origin of neurons distal to a SETS coaptation and thereby quantify the impact of a SETS transfer on native neuron regeneration. Fujiwara et al. (using a rat model with contralateral sciatic nerve to sciatic nerve SETS transfer to treat a sciatic nerve transection injury with ETE repair) found that the addition of a SETS transfer had no effect on the number of regenerating native motor or sensory neurons. ${ }^{14}$ However, the authors did acknowledge that the number of labeled neurons varied widely between each of their rats. In the setting of a crush injury, Nadi et al. used an elegant retrograde labeling technique to show that donor axons may have an inhibitory effect on native motor axon regeneration. ${ }^{16}$ The group reported that only $4 \%$ of native recipient motor axons had regenerated by 10 weeks after an injury treated with a SETS transfer compared to $12 \%$ after an injury without a SETS transfer.

Other studies from the peripheral nerve literature may give insights into how the presence of donor axons impacts native axon regeneration. Gordon et al. investigated whether side-to-side cross-bridge nerve grafts could be used to promote nerve regeneration through denervated distal nerve stumps. ${ }^{10,44}$ Although their focus was not the SETS transfer, they quantified motor axon regeneration from a donor nerve into a recipient nerve via a crossbridge graft in which the first coaptation (donor nerve to graft) was a traditional ETS transfer and the second coaptation (graft to recipient distal nerve stump) was in effect a SETS transfer. After delayed repair of the proximal recipient nerve, $100 \%$ of native recipient motor axons regenerated into a distal recipient stump protected by cross-bridge nerve grafts. ${ }^{10}$ The same authors showed that only $45 \%$ of native motor axons regenerated into a distal recipient stump after protection by a traditional ETS transfer ${ }^{11}$ and about $40 \%$ after delayed ETE repair without protection. ${ }^{10}$ They proposed that the amount of native recipient motor axon regeneration corresponds to the number of donor axons present in the recipient nerve-more donor axons means more recipient motor regeneration.

This effect may be mediated by the release of growth factors from the donor axons. Within 8 weeks of regaining axonal contact, Schwann cells revert to their mature myelinating phenotype, ${ }^{38}$ suggesting that persistence of an enriched regenerative environment in the recipient nerve beyond that period may not be attributable to Schwann cells. Gordon et al. found robust native axon regeneration into a distal nerve stump after ETE repair following 12 weeks of protection by donor axons via a cross-bridge graft. They hypothesized that a subset of donor axons within the recipient nerve is not in contact with Schwann cells and exhibits a sustained release of growth factors that potentiates native axon regeneration. ${ }^{10}$

Physical obstruction of regenerative pathways and motor end plates has been suggested as a mechanism by which donor axons could negatively impact native recipient regeneration. ${ }^{13}$ By virtue of being coapted more distally, donor axons may outcompete native axons from access to endoneurial structures and motor end plates. However, axons from different neurons do not usually withdraw from each other, ${ }^{45}$ making it less likely that physical obstruction restricts recipient axon access to subperineurial structures. Regenerating axons are also known to increase local expression of molecules capable of degrading 
growth-inhibiting proteoglycans ${ }^{46-48}$-if released by donor nerves, these molecules may make it physically easier for native axons to access distal endoneurial tubules. This "obstruction" hypothesis could be tested by eliminating donor pathways at various stages after a SETS coaptation as well as anterograde tracing strategies to reveal the percentage of motor end plates innervated by either recipient or donor axons.

Additionally, there is conflicting evidence as to whether protection by sensory fibers alone negatively ${ }^{15,43,49}$ or positively ${ }^{42}$ impacts native motor axon regeneration. This is clinically relevant, as multiple SETS transfers could be applied to a long nerve graft: a proximal SETS coaptation with a sensory donor nerve gives trophic support to regenerating native axons, while a distal motor SETS transfer prevents end-organ atrophy and augments function.

The impact of donor nerve size and fiber type, the mechanism of recipient nerve injury, the distance between recipient nerve injury and SETS coaptation as well as the timing of a recipient nerve repair are all material variables that have yet to be studied.

\section{End-Organ Influence on Axon Regeneration}

The importance of denervated muscle as a promoting factor for axonal regeneration through a denervated nerve stump is debated. ${ }^{50,51}$ In a SETS transfer, the influence of denervated muscle on regenerating donor axons may be negligible-previous work has shown that the same number of donor axons regenerated into a denervated stump after crossing a SETS coaptation regardless of whether the stump was isolated from denervated muscle. ${ }^{10}$ However, "target-derived" neurotrophic support seems to play a role in determining the specificity of axonal regeneration, ${ }^{52}$ with trophic support thought to diffuse through the nerve sheath in extracellular vesicles to reach regenerating axons. ${ }^{53}$ If donor axons reach muscle end-organ targets before regenerating recipient axons, it is possible that reinnervated muscle may alter its expression of neurotrophic factors, thereby causing a reduction in preferential recipient motor regeneration.

\section{Functional Outcomes After SETS Transfer Outcome Measures in Animal Models}

Functional outcomes after SETS transfer in animal studies have generally been reported as equal or superior to outcomes after ETE repair without SETS protection. ${ }^{6,13-15,17,19}$ Improved motor recovery after SETS transfer has most commonly been demonstrated using stimulated isolated muscle contraction force at sacrifice. ${ }^{6,13-15,17,19} \mathrm{Al}-$ though behavioral analysis has the benefit of serial in vivo testing, its role as a measure of integrated muscle function in the peroneal nerve to tibial nerve SETS hindlimb model may have inherent limitations. Nadi et al. were unable to demonstrate any functional effects in their model using a ladder rung analysis and noted that transfers between antagonistic nerve and muscle units can negatively impact functional performance. ${ }^{16,54}$ The use of an upper limb ulnar nerve to median nerve model in conjunction with weekly stimulated grip strength testing ${ }^{55}$ could overcome some of these limitations. The SETS transfer has been shown to increase muscle mass,${ }^{14}$ although this finding has not been consistent across different experimental models. ${ }^{9}, 16$

\section{Relative Functional Contribution of Donor Versus Recipient Axons}

The primary mechanism underlying improved muscle function after SETS transfer is not yet understood. Faster reinnervation of motor end plates by donor axons given the proximity of a SETS coaptation to muscle targets is believed to have a significant role. ${ }^{16,17} \mathrm{Up}$ to $90 \%$ of the total motor axon pool distal to a SETS transfer may originate from the donor nerve in the setting of a crush injury, raising the possibility that donor axons "outcompete" native axons to distal muscle targets. ${ }^{16}$

Conversely, if functional improvements were chiefly attributable to donor axons enriching the regenerative environment in the recipient nerve with consequently improved native motor regeneration, the beneficial impact of a SETS transfer on muscle atrophy might be less pronounced despite improved overall muscle force. ${ }^{19}$ In this scenario, regeneration of native axon motor units would account for the majority of muscle contraction force. This has been demonstrated experimentally by two groups. Fujiwara et al. reported a gastrocnemius muscle contraction force of $35 \pm 7.3$ on stimulation of the proximal recipient nerve compared to a force of $2.5 \pm 2.4$ after stimulating the proximal donor nerve ${ }^{14}$ whereas Farber et al. noted only a $25 \%$ decrease in evoked muscle force after dividing the SETS contribution. ${ }^{17}$ Other studies have found no differences in evoked muscle force after isolating stimulation to donor or recipient nerve pathways. ${ }^{13}$

The difficulty in assessing the mechanism of functional improvement after SETS transfers may be attributable to the use of rodent models with short experimental endpoints and short distances between the SETS transfer and proximal recipient nerve injury. Large animal models with greater regenerative distances could accentuate functional differences related either to early reinnervation of muscle targets by donor axons or to the impact of donor axons on native axon regeneration. These models would have the added benefit of being more reflective of clinical scenarios.

\section{Clinical Indications and Outcomes}

Proximal nerve injuries associated with delayed or incomplete end-organ reinnervation are indications for a SETS transfer. The most commonly used SETS transfer involves a distal AIN to ulnar nerve motor fascicle transfer to improve intrinsic hand function following proximal ulnar nerve injuries that would otherwise carry a poor prognosis..$^{18}$ Causes of ulnar neuropathy for which an AIN SETS transfer has been proposed include chronic compression secondary to cubital tunnel syndrome, brachial plexus traction injuries, brachial plexus neuritis as well as motor neuropathies and transection injuries between the proximal forearm and shoulder.

The published clinical experience with upper limb SETS transfers is limited to two retrospective case se$\operatorname{ries}^{8,56}$ and a retrospective comparative study. ${ }^{57}$ Davidge et al. performed AIN to ulnar motor SETS transfers in 55 patients with clinically significant ulnar intrinsic weakness 
TABLE 2. Recommendations for future research on the SETS transfer

\begin{tabular}{|c|c|}
\hline Mechanism & Question \\
\hline Entry of donor axons into recipient nerve & $\begin{array}{l}\text { 1. What depth of recipient connective tissue window maximizes donor axon growth across the } \\
\text { coaptation? }\end{array}$ \\
\hline Direction of donor axon growth & $\begin{array}{l}\text { 2. Does the initial direction of donor axon growth in the recipient nerve affect the impact of those } \\
\text { axons on muscle \& recipient axons? }\end{array}$ \\
\hline \multirow[t]{2}{*}{ Donor axon access to connective tissue structures } & 3a. Which factors regulate donor axon penetration into recipient perineurial structures? \\
\hline & $3 \mathrm{~b}$. What is the fate of axons regenerating outside of perineurial structures? \\
\hline \multirow[t]{5}{*}{ Native axon regeneration } & 4a. Does the presence of donor axons improve native axon regeneration? \\
\hline & 4b. How does the presence of donor axons affect native axon regeneration over time? \\
\hline & $\begin{array}{l}\text { 4c. What is the relative influence of Schwann cells vs the physical presence of donor axons on } \\
\text { native axon regeneration? }\end{array}$ \\
\hline & $\begin{array}{l}4 \mathrm{~d} \text {. Does donor nerve constitution (proportion of sensory vs motor axons) affect native motor } \\
\text { regeneration? }\end{array}$ \\
\hline & $\begin{array}{l}\text { 4e. Does recipient nerve injury mechanism (transection vs neuroma in continuity) affect native } \\
\text { axon regeneration after SETS? }\end{array}$ \\
\hline \multirow[t]{2}{*}{ End-organ influence } & 5a. Does denervated muscle provide trophic support for regenerating donor \& recipient axons? \\
\hline & $\begin{array}{l}\text { 5b. Does denervated muscle affect preferential motor regeneration differently in donor \& recipient } \\
\text { axon populations? }\end{array}$ \\
\hline \multirow[t]{2}{*}{ Relative contributions of donor \& recipient axons } & $\begin{array}{l}\text { 6a. What are the relative contributions of donor \& recipient axons to axon counts, motor end } \\
\text { plates, \& functional outcomes? }\end{array}$ \\
\hline & $6 \mathrm{~b}$. How do these relative contributions change over time? \\
\hline
\end{tabular}

mostly secondary to in-continuity lesions. ${ }^{8}$ They compared pre- and postoperative function using pinch and grip strengths, the Disabilities of the Arm, Shoulder, and Hand (DASH) score as well as manual muscle testing of the first dorsal interosseous muscle (Medical Research Council [MRC] grade) with a follow-up period of 12 months. Almost $50 \%$ of the patients had gradual improvement in intrinsic function between months 3 and 12 postoperatively. More than $70 \%$ scored grade 3 or higher on interosseous muscle strength per the MRC scale, with the greatest improvement in pinch and first interosseous muscle strength noted between the 1- to 3- and 3- to 6-month follow-up periods-earlier than would have been expected had nerve regeneration occurred solely via the native ulnar nerve. However, the authors could not ascertain the degree of improvement attributable to the SETS transfer given the risk of false positives in electrodiagnostic studies and highly subjective tests of intrinsic muscle function. They recommended that future trials adopt more robust assessments of intrinsic function and seek to provide direct evidence of recovery attributable to the SETS transfer, for example, through the use of targeted nerve blocks.

Baltzer et al. retrospectively compared the conventional surgical technique with AIN SETS transfer for the management of high ulnar nerve injuries. ${ }^{57}$ Their primary outcome measure was the clinically observed return of intrinsic function. The addition of a SETS transfer to manage ulnar transection injury resulted in a significantly greater return of intrinsic function than with conventional treatment ( $86 \%$ vs 14\%, 7 cases/group). Interestingly, there was no reported difference in the return of intrinsic function in patients with ulnar in-continuity lesions (6 cases/ group) - this finding echoes preclinical observations that a
SETS transfer may negatively impact regeneration across a neuroma-in-continuity injury. ${ }^{16}$

Other indirect evidence that donor axons from a SETS transfer contribute to functional recovery comes from two cases of AIN to ulnar nerve SETS transfers for severe cubital tunnel syndrome. ${ }^{56}$ These showed good motor recovery but a lack of sensory recovery, suggesting that distal nerve regeneration was largely attributable to the purely motor AIN SETS transfer rather than regenerating mixed ulnar nerve axons after decompression at the elbow. The SETS transfer has also been used successfully in singlestage facial reanimation procedures to augment smile function and symmetry after facial palsy. ${ }^{58-61}$

\section{Summary and Future Directions}

Whether the SETS transfer is ready for widespread clinical adoption given the material gaps in our understanding of its mechanisms (Table 2) has been the subject of controversy. ${ }^{7,12}$ There is consensus that robust donor nerve regeneration occurs across a SETS coaptation. These donor axons appear to have bidirectional growth upon entering the recipient nerve and are capable of forming motor units and reducing muscle atrophy. Technical considerations including optimal connective tissue window depth have yet to be studied. The impact of muscle end organ, donor axons, and donor fiber type on the regenerative potential of native recipient axons is poorly understood. There is limited evidence that the mechanism of recipient nerve injury affects the relative contributions of donor and recipient axons to distal axon counts and muscle function.

A better understanding of this transfer's mechanisms with subsequent technical optimization is key to unlock- 
ing its clinical potential. Improved animal models and prospective clinical studies with reliable outcome assessments will help to delineate the indications and impact of SETS transfers in clinical scenarios.

\section{References}

1. Höke A. Mechanisms of disease: what factors limit the success of peripheral nerve regeneration in humans? Nat Clin Pract Neurol. 2006;2(8):448-454.

2. Fu SY, Gordon T. Contributing factors to poor functional recovery after delayed nerve repair: prolonged denervation. $J$ Neurosci. 1995;15(5 pt 2):3886-3895.

3. Gordon T, Tyreman N, Raji MA. The basis for diminished functional recovery after delayed peripheral nerve repair. $J$ Neurosci. 2011;31(14):5325-5334.

4. Sulaiman OA, Gordon T. Effects of short- and long-term Schwann cell denervation on peripheral nerve regeneration, myelination, and size. Glia. 2000;32(3):234-246.

5. Tung TH, Mackinnon SE. Nerve transfers: indications, techniques, and outcomes. J Hand Surg Am. 2010;35(2):332-341.

6. Isaacs J, Allen D, Chen LE, Nunley J II. Reverse end-to-side neurotization. J Reconstr Microsurg. 2005;21(1):43-50.

7. Isaacs J. Supercharged end-to-side nerve transfer: too soon for "prime time"? J Hand Surg Am. 2013;38(3):617-618.

8. Davidge KM, Yee A, Moore AM, Mackinnon SE. The supercharge end-to-side anterior interosseous-to-ulnar motor nerve transfer for restoring intrinsic function: clinical experience. Plast Reconstr Surg. 2015;136(3):344e-352e.

9. Kale SS, Glaus SW, Yee A, et al. Reverse end-to-side nerve transfer: from animal model to clinical use. J Hand Surg Am. 2011;36(10):1631-1639.e2.

10. Gordon T, Hendry M, Lafontaine CA, et al. Nerve crossbridging to enhance nerve regeneration in a rat model of delayed nerve repair. PLoS One. 2015;10(5):e0127397.

11. Sulaiman OAR, Gordon T. A rat study of the use of end-toside peripheral nerve repair as a "babysitting" technique to reduce the deleterious effect of chronic denervation. J Neurosurg. 2018;131(2):622-632.

12. Mackinnon SE. In reply. J Hand Surg Am. 2013;38(3):618619.

13. Isaacs JE, Cheatham S, Gagnon EB, et al. Reverse end-toside neurotization in a regenerating nerve. J Reconstr Microsurg. 2008;24(7):489-496.

14. Fujiwara T, Matsuda K, Kubo T, et al. Axonal supercharging technique using reverse end-to-side neurorrhaphy in peripheral nerve repair: an experimental study in the rat model. $J$ Neurosurg. 2007;107(4):821-829.

15. Li Q, Zhang P, Yin X, et al. Early sensory protection in reverse end-to-side neurorrhaphy to improve the functional recovery of chronically denervated muscle in rat: a pilot study. J Neurosurg. 2014;121(2):415-422.

16. Nadi M, Ramachandran S, Islam A, et al. Testing the effectiveness and the contribution of experimental supercharge (reversed) end-to-side nerve transfer. J Neurosurg. 2018;130(3):702-711.

17. Farber SJ, Glaus SW, Moore AM, et al. Supercharge nerve transfer to enhance motor recovery: a laboratory study. $J$ Hand Surg Am. 2013;38(3):466-477.

18. Barbour J, Yee A, Kahn LC, Mackinnon SE. Supercharged end-to-side anterior interosseous to ulnar motor nerve transfer for intrinsic musculature reinnervation. J Hand Surg Am. 2012;37(10):2150-2159.

19. Isaacs J, Patel G, Mallu S, et al. Effect of reverse endto-side (supercharging) neurotization in long processed acellular nerve allograft in a rat model. J Hand Surg Am. 2019;44(5):419.e1-419.e10.
20. Balik O, Menderes A. A successful neurotization of two different muscles using a single intact motor nerve: experimental study on rats. Ann Plast Surg. 2011;66(2):172-178.

21. Brushart TM. Motor axons preferentially reinnervate motor pathways. J Neurosci. 1993;13(6):2730-2738.

22. Madison RD, Archibald SJ, Brushart TM. Reinnervation accuracy of the rat femoral nerve by motor and sensory neurons. J Neurosci. 1996;16(18):5698-5703.

23. Madison RD, Robinson GA, Chadaram SR. The specificity of motor neurone regeneration (preferential reinnervation). Acta Physiol (Oxf). 2007;189:201-206.

24. Zhang ZY, Li FC, Shao M, et al. Allogeneic hand transplantation and rehabilitation of hand function: a 10-year followup study. Int Wound J. 2016;13(6):1303-1308.

25. Karacaoglu E, Yüksel F, Peker F, Güler MM. Nerve regeneration through an epineurial sheath: its functional aspect compared with nerve and vein grafts. Microsurgery. 2001;21(5):196-201.

26. Łukaszuk M, Kwiecień G, Madajka M, et al. Repair of the peripheral nerve gap with epineural sheath conduit to prevent muscle denervation atrophy in the diabetic rat model. Pol Przegl Chir. 2013;85(7):387-394.

27. Barmpitsioti A, Konofaos P, Ignatiadis I, et al. Nerve growth factor combined with an epineural conduit for bridging a short nerve gap $(10 \mathrm{~mm})$. A study in rabbits. Microsurgery. 2011;31(7):545-550.

28. Livet J, Weissman TA, Kang H, et al. Transgenic strategies for combinatorial expression of fluorescent proteins in the nervous system. Nature. 2007;450(7166):56-62.

29. Sulaiman W, Gordon T. Neurobiology of peripheral nerve injury, regeneration, and functional recovery: from bench top research to bedside application. Ochsner J. 2013;13(1):100108.

30. Scheib J, Höke A. Advances in peripheral nerve regeneration. Nat Rev Neurol. 2013;9(12):668-676.

31. Zochodne DW. The challenges and beauty of peripheral nerve regrowth. J Peripher Nerv Syst. 2012;17(1):1-18.

32. Weinberg HJ, Spencer PS. The fate of Schwann cells isolated from axonal contact. J Neurocytol. 1978;7(5):555-569.

33. Giannini C, Dyck PJ. The fate of Schwann cell basement membranes in permanently transected nerves. J Neuropathol Exp Neurol. 1990;49(6):550-563.

34. Siironen J, Vuorinen V, Taskinen HS, Röyttä M. Axonal regeneration into chronically denervated distal stump. 2. Active expression of type I collagen mRNA in epineurium. Acta Neuropathol. 1995;89(3):219-226.

35. Höke A, Gordon T, Zochodne DW, Sulaiman OAR. A decline in glial cell-line-derived neurotrophic factor expression is associated with impaired regeneration after long-term Schwann cell denervation. Exp Neurol. 2002;173(1):77-85.

36. You S, Petrov T, Chung PH, Gordon T. The expression of the low affinity nerve growth factor receptor in long-term denervated Schwann cells. Glia. 1997;20(2):87-100.

37. Ronchi G, Cillino M, Gambarotta G, et al. Irreversible changes occurring in long-term denervated Schwann cells affect delayed nerve repair. J Neurosurg. 2017;127(4):843-856.

38. Hendry JM, Alvarez-Veronesi MC, Snyder-Warwick A, et al. Side-to-side nerve bridges support donor axon regeneration into chronically denervated nerves and are associated with characteristic changes in Schwann cell phenotype. Neurosurgery. 2015;77(5):803-813.

39. Pellegrino RG, Spencer PS. Schwann cell mitosis in response to regenerating peripheral axons in vivo. Brain Res. 1985;341(1):16-25.

40. Li H, Terenghi G, Hall SM. Effects of delayed re-innervation on the expression of c-erbB receptors by chronically denervated rat Schwann cells in vivo. Glia. 1997;20(4):333-347.

41. Carroll SL, Miller ML, Frohnert PW, et al. Expression of neuregulins and their putative receptors, ErbB2 and ErbB3, 
is induced during Wallerian degeneration. J Neurosci. 1997; 17(5):1642-1659.

42. Placheta E, Wood MD, Lafontaine C, et al. Enhancement of facial nerve motoneuron regeneration through cross-face nerve grafts by adding end-to-side sensory axons. Plast Reconstr Surg. 2015;135(2):460-471.

43. Li QT, Zhang PX, Yin XF, et al. Functional recovery of denervated skeletal muscle with sensory or mixed nerve protection: a pilot study. PLoS One. 2013;8(11):e79746.

44. Ladak A, Schembri P, Olson J, et al. Side-to-side nerve grafts sustain chronically denervated peripheral nerve pathways during axon regeneration and result in improved functional reinnervation. Neurosurgery. 2011;68(6):1654-1666.

45. Kang H, Lichtman JW. Motor axon regeneration and muscle reinnervation in young adult and aged animals. J Neurosci. 2013;33(50):19480-19491.

46. Zuo J, Ferguson TA, Hernandez YJ, et al. Neuronal matrix metalloproteinase-2 degrades and inactivates a neuriteinhibiting chondroitin sulfate proteoglycan. J Neurosci. 1998;18(14):5203-5211.

47. Zuo J, Neubauer D, Graham J, et al. Regeneration of axons after nerve transection repair is enhanced by degradation of chondroitin sulfate proteoglycan. Exp Neurol. 2002;176(1):221-228.

48. La Fleur M, Underwood JL, Rappolee DA, Werb Z. Basement membrane and repair of injury to peripheral nerve: defining a potential role for macrophages, matrix metalloproteinases, and tissue inhibitor of metalloproteinases-1. J Exp Med. 1996;184(6):2311-2326.

49. Sulaiman OAR, Midha R, Munro CA, et al. Chronic Schwann cell denervation and the presence of a sensory nerve reduce motor axonal regeneration. Exp Neurol. 2002;176(2):342-354.

50. Frey M, Koller R, Liegl C, et al. Role of a muscle target organ on the regeneration of motor nerve fibres in long nerve grafts: a synopsis of experimental and clinical data. Microsurgery. 1996;17(2):80-88.

51. Chen YG, Brushart TM. The effect of denervated muscle and Schwann cells on axon collateral sprouting. J Hand Surg Am. 1998;23(6):1025-1033.

52. Madison RD, Sofroniew MV, Robinson GA. Schwann cell influence on motor neuron regeneration accuracy. Neuroscience. 2009;163(1):213-221.

53. Madison RD, Robinson GA. Accuracy of regenerating motor neurons: influence of diffusion in denervated nerve. Neuroscience. 2014;273:128-140.

54. Lutz BS, Chuang DCC, Hsu JC, et al. Selection of donor nerves - an important factor in end-to-side neurorrhaphy. $\mathrm{Br}$ J Plast Surg. 2000;53(2):149-154.
55. Hanwright PJ, Rath JL, von Guionneau N, et al. Stimulated grip strength measurement: validation of a novel method for functional assessment. Muscle Nerve. 2019;60(4):437-442.

56. Jarvie G, Hupin-Debeurme M, Glaris Z, Daneshvar P. Supercharge end-to-side anterior interosseous nerve to ulnar motor nerve transfer for severe ulnar neuropathy: two cases suggesting recovery secondary to nerve transfer. J Orthop Case Rep. 2018;8(5):25-28.

57. Baltzer H, Woo A, Oh C, Moran SL. Comparison of ulnar intrinsic function following supercharge end-to-side anterior interosseous-to-ulnar motor nerve transfer: a matched cohort study of proximal ulnar nerve injury patients. Plast Reconstr Surg. 2016;138(6):1264-1272.

58. Frey M, Giovanoli P, Michaelidou M. Functional upgrading of partially recovered facial palsy by cross-face nerve grafting with distal end-to-side neurorrhaphy. Plast Reconstr Surg. 2006;117(2):597-608.

59. Sforza C, Frigerio A, Mapelli A, et al. Double-powered free gracilis muscle transfer for smile reanimation: a longitudinal optoelectronic study. J Plast Reconstr Aesthet Surg. 2015;68(7):930-939.

60. Biglioli F, Colombo V, Tarabbia F, et al. Double innervation in free-flap surgery for long-standing facial paralysis. J Plast Reconstr Aesthet Surg. 2012;65(10):1343-1349.

61. Vachata P, Brusakova S, Lodin J, Sames M. Masseteric nerve supercharge bypass in primary reconstruction of facial nerve. Acta Neurochir (Wien). 2019;161(6):1089-1094.

\section{Disclosures}

Dr. Brandacher has direct stock ownership in Ossium Health and is a medical advisor for X-Therma Inc.

\section{Author Contributions}

Conception and design: von Guionneau, Tuffaha. Acquisition of data: von Guionneau. Analysis and interpretation of data: von Guionneau, Tuffaha. Drafting the article: von Guionneau. Critically revising the article: all authors. Reviewed submitted version of manuscript: all authors. Approved the final version of the manuscript on behalf of all authors: Belzberg. Study supervision: Tuffaha.

\section{Correspondence}

Allan Belzberg: Johns Hopkins University School of Medicine, Baltimore, MD. abelzbe1@jhmi.edu. 